\title{
Interleukin-17 inhibitors. A new era in treatment of psoriasis and other skin diseases
}

\author{
Agnieszka Wasilewska, Marta Winiarska, Małgorzata Olszewska, Lidia Rudnicka
}

Department of Dermatology, Medical University of Warsaw, Warsaw, Poland

Adv Dermatol Allergol 2016; XXXIII (4): 247-252

DOI: 10.5114/ada.2016.61599

\begin{abstract}
Psoriasis is a chronic skin disease caused by the excessive secretion of inflammatory cytokines. Available therapeutic options include biologic drugs such as tumor necrosis factor alpha inhibitors and interleukin 12/23 (IL-12/23) inhibitors. The recent discovery of IL-17, which contributes to development of psoriasis, opened new possibilities for further treatment modalities. Currently, one anti-IL17 biological agent is approved for the treatment - a fully human monoclonal antibody that targets IL-17A (secukinumab). Further clinical trials, including a humanized IgG4 specific for IL-17 (ixekizumab) and a fully human antibody that targets the IL-17 receptor A (brodalumab).
\end{abstract}

Key words: alopecia areata, fynomer, lichen planus, pemphigoid, pemphigus.

\section{Introduction}

Psoriasis is a chronic inflammatory skin disease affecting about $2 \%$ of the Western population $[1,2]$. It presents as erythematous scaly plaques with induration. Psoriasis can affect the patient's quality of life and self-esteem $[3,4]$. Topical treatment includes application of coal tar, dithranol, retinoids, corticosteroids and vitamin $\mathrm{D}_{3}$ analogues. Systemic treatment includes phototherapy, methotrexate, cyclosporine, retinoids and biological agents. Despite many options, many patients do not respond to therapy or report side effects. Thus, new drugs are still being searched for.

\section{Interleukin-17 and pathogenesis of psoriasis}

Thelper cells have been classically divided into Th1 and Th2. In 2005, a new class of Thelper cells (Th17) was discovered [5]. It was demonstrated that Th17 cells play a role in the protection from both extracellular and intracellular agents [6]. Transforming growth factor (TGF)- $\beta$ and interleukin (IL)-6 stimulate the differentiation of naïve CD4+ T cells into Th17. This induces the expression of IL-17A [7]. IL-17A is a pro-inflammatory cytokine. It belongs to the IL-17 family, which consists of IL-17A-F [8]. IL-17A plays a role in neutrophil recruitment, host defense and immuno-inflammatory pathology [7]. It is secreted mainly by Th17, but also by Treg cells, NK cells, mast cells and neutrophils [9]. IL-17A and IL-17F bind to the same receptor, however the influence of IL-17A on gene regulation is 10-30 times stronger. The function of IL-17B, IL-17C and IL-17D is poorly defined. IL-17E limits Th17 development and promotes Th2 cytokines [10]. IL-17 plays a role in numerous immune-mediated disorders, such as rheumatoid arthritis, Crohn's disease, multiple sclerosis and autoimmune encephalomyelitis [11-13]. The role of IL-17A in atherosclerosis is under discussion. Studies showed both its proatherogenic and protective influence [14].

It is believed that an unknown antigen triggers NK cells, plasmacytoid dendritic cells and macrophages to secrete tumor necrosis factor (TNF)- $\alpha$, IL-1 $1 \beta$, interferon (IFN)- $\alpha$ and IL-6 in genetically susceptible patients. Local myeloid dendritic cells, activated during this process, produce IL-12 and IL-23 [15]. IL-23 plays a role in the maintenance of Th17 response. IL-12, on the other hand, upregulates the proliferation of Th1 cells. IL-17A upregulates the keratinocyte chemokine CCL2O, and recruits CCR6+ cells ( $\mathrm{mDCs}$ and Th17). Moreover, it induces the expression of neutrophil chemoattractant chemokines and antimicrobial peptides [7, 11, 16]. IL-17A sustains chronic inflammations [17].

\section{Interleukin-17 inhibitors}

As a result of the success of biological treatment targeting the IL-23/Th17 pathway, investigators still search

Address for correspondence: Prof. Lidia Rudnicka MD, PhD, Department of Dermatology, Medical University of Warsaw, 92a Koszykowa St, 02-008 Warsaw, Poland, phone: +48 22502 13 24, e-mail: lidiarudnicka@gmail.com Received: 17.07.2015, accepted: 6.01.2016. 
for new therapeutic options. Three agents that block IL17 are investigated at the moment. Secukinumab, brodalumab and ixekizumab have successfully completed phase II clinical trial and are currently at phase III [11]. The results are very promising.

\section{Secukinumab (AIN457)}

Secukinumab is a fully human anti-IL-17A monoclonal antibody. Secukinumab showed efficacy in the treatment of moderate-to-severe plaque psoriasis in a randomized double-blind placebo-controlled phase $I$ regimen finding study. In the study, the patients were randomized to a placebo group, a single drug administration group, an early group (at weeks $0,1,2,4$ ) or a monthly group (at weeks $0,4,8$ ). At week 12, the early and monthly induction regimens resulted in higher PASI75 response rates vs. placebo (54.5\% and $42.0 \%$ vs. $1.5 \%$; $p<0.001$ for both). After 12 weeks, patients who achieved Psoriasis Area Severity Index (PASI)75 were re-randomized to a secukinumab fixed interval regimen (150 mg at weeks 12 or 24) or a treatment-at-start-of-relapse maintenance regimen. Between weeks 20 and 28, PASI75 or PASI90 were more frequently achieved in the fixed interval group than with the fixed interval regimen $(85 \%$ and $58 \%$ vs. $67 \%$ and $21 \%$, respectively) [18].

In two phase III, double blind, 52-week trials, ERASURE (Efficacy of Response and Safety of Two Fixed Secukinumab Regimens in Psoriasis) and FIXTURE (Full Year Investigative Examination of Secukinumab vs. Etanercept Using Two Dosing Regimens to Determine Efficacy in Psoriasis), the efficacy of secukinumab was investigated in patients with moderate-to-severe plaque psoriasis. The ERASURE study included 738 patients, and the FIXTURE one - 1306 patients. Both groups received either placebo or secukinumab subcutaneously once a week for 5 weeks, then once a month. Patients in the FIXTURE study were also given etanercept of $50 \mathrm{mg}$ twice a week for 12 weeks, then once a week.

In the ERASURE study, PASI75 at week 12 was achieved by $81.6 \%$ and $71.6 \%$ of patients administered with $300 \mathrm{mg}$ and $150 \mathrm{mg}$ of secukinumab, respectively and $4.5 \%$ of placebo patients. In the FIXTURE study, $77.1 \%$ of patients administered with $300 \mathrm{mg}$ of secukinumab, $67 \%$ of those administered with $150 \mathrm{mg}$ of secukinumab, $44 \%$ of etanercept patients and only $4.9 \%$ of placebo patients achieved PASI75 at week 12. In the ERASURE study, the percentage of patients who got a response of 0 or 1 in the modified Investigator's Global Assessment at week 12 was $65.3 \%, 51.2 \%$, and $2.4 \%$ among patients who received secukinumab of $300 \mathrm{mg}, 150 \mathrm{mg}$ and placebo, respectively; in the FIXTURE study the rates were $62.5 \%$ with $300 \mathrm{mg}$ of secukinumab, $51.1 \%$ with $150 \mathrm{mg}$ of secukinumab, $27.2 \%$ with etanercept, and $2.8 \%$ with placebo ( $p<0.001$ for each secukinumab dose vs. comparators). Adverse effects in the ERASURE study were more common in the secukinumab group than in the placebo group and mostly included nasopharyngitis, headache and upper respiratory tract infections. In the FIXTURE study, the incidence of adverse effects was similar among secukinumab and etanercept patients. The most common side effects were nasopharyngitis, headache and diarrhea [19].

A 24-week, randomized, double blind, placebo-controlled, phase II proof-of-concept trial included $42 \mathrm{pa}$ tients with moderate-to-severe psoriatic arthritis who met CASPAR criteria. Twenty-eight patients were administered with two intravenous secukinumab doses of $10 \mathrm{mg} / \mathrm{kg}$ every 3 weeks and 14 patients were administered with placebo. The primary endpoint was ACR20 responses at week 6 . The results were $39 \%$ in secukinumab vs. $23 \%$ in placebo patients. The respective results for weeks 12 and 24 were $39 \%$ vs. $15 \%$ and $43 \%$ vs. $18 \%$. Two patients from the secukinumab group dropped out of the study due to withdrawal of consent and 1 due to the unsatisfactory therapeutic effect. In the placebo group, 3 patients dropped out of the study due to the withdrawal of consent and 1 had an unsatisfactory effect. The most common adverse effects included nasopharyngitis, headache, nausea, dizziness, fatigue and diarrhea [20].

Self-administration of secukinumab via sc route was safe and more effective than placebo. At week 12, PASI75 was achieved by $75.9 \%, 69.5 \%$, and $0 \%$ of patients who were administered with secukinumab of 300 mg, 150 mg and placebo, respectively [21].

The evaluation of the safety, tolerability and efficacy of secukinumab is being carried out in an ongoing clinical trial FUTURE 1. The study includes patients with active psoriatic arthritis who did not tolerate or were irresponsive to nonsteroidal anti-inflammatory drugs (NSAIDs), DMARDs and/or TNF- $\alpha$ inhibitor therapy. It evaluates patients treated with $75 \mathrm{mg}$ or $150 \mathrm{mg}$ of secukinumab vs. placebo who achieved ACR20. The study is expected to be completed in November 2014 (Clinicaltrials.gov identifier NCT01392326).

Another ongoing study is a study on safety, tolerability, and efficacy of secukinumab in subjects with moderate-to-severe nail psoriasis (TRANSFIGURE) (ClinicalTrials. gov identifier NCT01807520).

In phase III randomized, double-blind, placebo-controlled multicenter study, secukinumab is being evaluated in patients with moderate-to-severe palmoplantar psoriasis. The results will be available in November 2015 (ClinicalTrials.gov identifier NCT01806597).

\section{Brodalumab (AMG 827)}

Brodalumab is a human, anti-IL17RA monoclonal antibody. It blocks the activity of IL17RA, 17A/F and 17E.

Russell et al. reported that IL-17R blockade with brodalumab normalizes the psoriasis transcriptome. Keratinocyte expressed genes, e.g. KRT6A, IL1F6, chemokine CXCL6, antimicrobial peptide gene S100A7A normalize 
faster than T cell genes. Ki67 cell counts returned to normal after 2 weeks, but the inflammatory leukocyte infiltrate decreased after 6 weeks [22].

In a placebo-controlled phase I study, 25 patients with moderate-to-severe psoriasis received either a single dose of brodalumab: $140 \mathrm{mg}$ s.c. $(n=4), 350 \mathrm{mg}$ s.c. $(n=8)$, $700 \mathrm{mg}$ i.v. $(n=8)$, or placebo $(n=5)$. All the patients who received brodalumab $700 \mathrm{mg}$ i.v. achieved PASI50 by day 29, 7 of them had PASI75 by Day 43 and 3 patients achieved PASI90 by day 43. In the $350 \mathrm{mg}$ brodalumab sc group, 6 patients achieved PASI50 and 3 patients PASI75. Only 2 patients in the $140 \mathrm{mg}$ brodalumab group achieved PASI50, and none in the placebo group. The $350 \mathrm{mg}$ S.c. and $700 \mathrm{mg}$ i.v. brodalumab groups also showed a reduction in epidermal thickening and keratin 16 levels and a significant improvement in mRNA levels of IL-17 modulated keratinocyte-derived factors, e.g. DEFB4, cathelicidin, KRT 16, CCL18, CCL20. Moreover, cytokines not directly regulated by IL-17R, (IL-22, IL-23) were reduced. The levels of IL-17A, IL-17C and IL-17F were similar to those in healthy individuals. The safety of brodalumab was similar to placebo [23].

In phase II, randomized, double-blind, placebo-controlled, dose-ranging study in moderate-to-severe plaque psoriasis, brodalumab proved to be effective. One hundred and ninety-eight patients received either placebo or the following doses of brodalumab: $70 \mathrm{mg}, 140 \mathrm{mg}$, $210 \mathrm{mg}$ on day 1 and at weeks 1, 2, 4, 6, 8 and 10. The last group received $280 \mathrm{mg}$ of brodalumab on day 1 and at weeks 4 and 8 . At week 12 the improvement in PASI was $85.9 \%$, 86.3\%, 76\%, 45\% in $140 \mathrm{mg}, 210 \mathrm{mg}, 280 \mathrm{mg}$, $70 \mathrm{mg}$ brodalumab groups, respectively, versus $16 \%$ in the placebo group. At week 12 PASI75 and PASI90 were observed in $77 \%$ and $72 \%$, respectively, in patients of $140 \mathrm{mg}$ brodalumab group and $82 \%$ and $75 \%$, respectively, in patients of $210 \mathrm{mg}$ brodalumab group in comparison with $0 \%$ in the placebo group. The most common adverse events in brodalumab groups were nasopharyngitis, upper respiratory tract infection and injection-site erythema. There were 2 cases of grade 3 neutropenia which resolved with the discontinuation of the drug [24].

In phase 1 randomized double-blind placebocontrolled study 168 patients with psoriatic arthritis were randomized to brodalumab $140 \mathrm{mg}$, brodalumab $280 \mathrm{mg}$ or a placebo group. The drug was administered on day 1 and at weeks 1, 2, 4, 6, 8 and 10. After 12 weeks, the patients who did not discontinue the study, were offered open label brodalumab 280 mg every 2 weeks. At week $12,37 \%, 39 \%$ and $18 \%$ of patients met American College of Rheumatology response criteria (ACR20) in brodalumab $140 \mathrm{mg}$, brodalumab $280 \mathrm{mg}$ and placebo groups, respectively. Moreover, patients from both brodalumab groups had a higher ACR50 response in comparison with placebo (14\% vs. $4 \%$ ). At week 24 , ACR20 was achieved in $51 \%$ and $64 \%$ of patients in brodalumab $140 \mathrm{mg}$ and $280 \mathrm{mg}$ groups, respectively, and $44 \%$ of pa- tients who switched from placebo to open label brodalumab group. Adverse events occurred in $62 \%, 71 \%$ and $65 \%$ in brodalumab $140 \mathrm{mg}, 280 \mathrm{mg}$ and placebo groups, respectively. The most common adverse effects were the infections of the upper respiratory tract, fatigue, diarrhea and headache. Serious adverse events, which occurred in four patients, included abdominal pain, cholecystitis and cellulitis on the knee and the upper chest [25].

In two phase-3 studies (AMAGINE 2 and AMAGINE 3) brodalumab at doses of $140 \mathrm{mg}$ S.C. and $210 \mathrm{mg}$ S.C. was compared with ustekinumab and placebo in patients with moderate-to-severe plaque psoriasis. At week 12 patients who received brodalumab were re-randomized to receive brodalumab of $210 \mathrm{mg}$ every 2 weeks or $140 \mathrm{mg}$ every 2, 4 or 8 weeks. Patients in ustekinumab continued with ustekinumab and patients in the placebo group received brodalumab of $210 \mathrm{mg}$ every 2 weeks. At week 12 , PASI 75 was achieved in $86 \%$ and $67 \%$ patients in the brodalumab group $210 \mathrm{mg}$ and $140 \mathrm{mg}$ (AMAGINE-2), respectively, and $85 \%$ and $69 \%$ in AMAGINE-3. PASI75 was achieved by $70 \%$ and $69 \%$ of patients in the ustekinumab group, AMAGINE-2 and AMAGINE-3, respectively. PASI100 was achieved by 44\% (AMAGINE-2) and $37 \%$ (AMAGINE-3) of patients in brodalumab $210 \mathrm{mg}$ group and 22\% (AMAGINE-2) and 19\% (AMAGINE-3) in the ustekinumab group. At week 52 the best results were seen in patients who received $210 \mathrm{mg}$ of brodalumab every 2 weeks. In AMAGINE-2, 63\% of patients score 0 or 1 in SPGA and $61 \%$ in AMAGINE-3. The adverse effects were more frequent in the brodalumab group than in the ustekinumab group. The most common adverse effect included nasopharyngitis, arthralgia, headache and upper respiratory tract infections and Candida infections. There were reported cases of death among patients in the brodalumab group - due to stroke, cardiac arrest, carcinoma of pancreas, cardiomyopathy and hematophagic histiocytosis syndrome. Two deaths were due to suicides [26].

In another phase-III randomized, double-blind, placebo-controlled study, the safety and efficacy of brodalumab in comparison with placebo at week 16 are being evaluated in patients with psoriatic arthritis. The results of the study are expected to be available in April 2018 (ClinicalTrials.gov Identifier NCT02024646).

In 2015, Amgen ended the participation in the project related to brodalumab because of two deaths due to suicide. The project is continued by AstraZeneca.

\section{Ixekizumab (LY2439821)}

Ixekizumab is a humanized IgG4 monoclonal antibody that neutralizes IL-17 [27].

In a 20-week randomized double-blind placebo-controlled phase I study, ixekizumab was evaluated in 40 individuals with chronic moderate-to-severe plaque psoriasis. Patients received $5 \mathrm{mg}, 15 \mathrm{mg}, 50 \mathrm{mg}$ or $150 \mathrm{mg}$ of ixekizumab sc or placebo at weeks 0, 2 and 4. Punch biopsies were performed at weeks 0, 2 and 6. At week 2 
there were a reduction in keratinocyte proliferation, epidermal hyperplasia, dermal infiltration of T cells and dendritic cells and keratinocyte expression of innate defense peptides. The reduction was dependent on the dosage. At week 6 the skin was normal [28].

In phase II, double-blind, placebo-controlled trial, 142 patients with moderate-to-severe plaque psoriasis were randomized to receive $10,25,75,150 \mathrm{mg}$ of ixekizumab s.c. or placebo at weeks 0, 2, 4, 8, 12 and 16. At week $12,76.7 \%, 82.8 \%$ and $82.1 \%$ of patients achieved PASI75 in groups: $25 \mathrm{mg}, 75 \mathrm{mg}, 150 \mathrm{mg}$ of ixekizum$\mathrm{ab}$, respectively, versus $7.7 \%(p<0.001)$ in the placebo group. More patients treated with a high dosage of ix ekizumab at week 12 achieved PASI90 (150 mg - 71.4\%, $75 \mathrm{mg}-58.6 \%, 25 \mathrm{mg}-50 \%$, placebo - 0\%) and PASI100 (150 mg-39.3\%, $75 \mathrm{mg}-37.9 \%)$. The side effects included nasopharyngitis, upper respiratory tract infections, injection site reactions and headache. Two patients receiving ixekizumab had grade 2 neutropenia according to the Common Terminology Criteria for Adverse Events. Four patients dropped out of the study because of adverse effects: hypertriglyceridemia, peripheral edema, hypersensitivity, and urticaria [29].

A post-hoc analysis conducted by Zhu et al. proved that with an early clinical improvement in PASI we can predict the PASI response when treating psoriasis patients with ixekizumab. According to the study, achieving PASI40 at weeks 4 or 6 was associated with high negative predictive values ( $80 \%$ and $95 \%$, respectively) and positive predictive values ( $89 \%$ and $84 \%$, respectively). Achieving PASI50 showed more accuracy - negative predictive values (NPVs) of $71 \%$ and $89 \%$ and positive predictive values (PPVs) of $94 \%$ and $89 \%$, respectively. Patients who do not achieve PASI40 at weeks 4-6 are unlikely to respond ixekizumab therapy. Since biological treatment is very costly, these findings may be very helpful to predict therapy results and further investigation needs to be conducted [30].

The efficacy and safety of ixekizumab were investigated in a 52-week open-label study in patients with chronic plaque psoriasis. Patients received $10 \mathrm{mg}, 25 \mathrm{mg}$, $75 \mathrm{mg}$ or $150 \mathrm{mg}$ of ixekizumab at weeks 0, 2, 4, 8, 12 and 16 or placebo. At week 20, patients who did not achieve PASI75 were switched to open label extensions (OLE) and received $120 \mathrm{mg}$ S.c. of ixekizumab every 4 weeks. Patients who achieved PASI75 entered a treatment-free period between weeks 20 and 32, then entered the open label extension after meeting the criteria. Those who completed the randomized double-blind clinical trial entered the open label extension. Fifty-eight percent of them achieved PASI75 at week 20 and entered a treatment-free period at weeks 20-32. Thirty-five percent lost PASI75 within this period and entered the OLE. Patients who did not respond to ixekizumab at week 20 entered the OLE. At week 52, $77 \%$ of those who entered the OLE achieved PASI75, 68\% achieved PASI90 and 48\% - PASI100. Among patients who had PASI75 at week 20, 95\% still had PASI75 at week 52. In patients who achieved PASI90 or PASI100 at week 20, the respective PASI rates were $94 \%$ and $82 \%$. The initial dosage of ixekizumab was not important for the results at week 52 and the improvement was similar in each group. Serious adverse effects observed in 10 patients included rectal cancer, hidradenitis suppurativa, depression, suicide attempt, atherosclerosis, congestive heart failure, urinary tract obstruction, cellulitis, pyelonephritis, acute coronary syndrome, nephrolithiasis, wrist fracture and laceration of the arm. No cases of neutropenia higher than grade 2 were reported [31].

There is an ongoing multicenter, randomized, doubleblind, active (with adalimumab) and placebo-controlled study which investigates the efficacy and safety of ixekizumab in patients with psoriatic arthritis (ClinicalTrials. gov identifier NCT01695239).

The results of phase III study comparing the efficacy and safety of ixekizumab to etanercept and placebo in patients with moderate-to-severe plaque psoriasis will be known in December 2018 (ClinicalTrials.gov Identifier NCT01597245).

\section{Novel drug candidates targeting IL-17}

Several agents which target the IL-17-TH17 pathway are currently under investigation [31, 32]. The ongoing studies focus on the efficacy of anti-IL-17 antibodies (bimekizumab, ALX-0761, CJM112, CNTO 6785, LY3074828, and SCH-900117). A novel approach is the application of anti-IL-17 nanoantibodies (e.g. MSB0010841) in treatment of psoriasis. Dual anti IL-17/TNF- $\alpha$ inhibitors (e.g. ABT-122, COVA322) are currently in early phases of clinical trials. Fynomers are small binding proteins engineered to target molecules with the same affinity and specificity as antibodies. Genetic fusion of fynomers to antibodies allows the production of a bi-specific, fully human antiTNF and anti-IL-17A antibody (FynomAb ${ }^{\oplus}$ COVA322) [33].

\section{Agents targeting IL-17 beyond psoriasis}

Several pre-clinical data indicate that IL-17 inhibitors may be effective in multiple muco-cutaneous disorders beyond psoriasis. The possible targets for IL-17 inhibitors include oral lichen planus [34], alopecia areata [35], pyoderma gangrenosum [36], palmo-plantar pustulosis [36], systemic lupus erythematosus [37], systemic sclerosis [37], mixed connective tissue disease [37], pemphigus vulgaris [38], pemphigoid [38, 39], dermatitis herpetiformis [39], atopic dermatitis [40] and chronic periodontitis [41].

\section{Conclusions}

Psoriasis is a chronic inflammatory disease. Despite many options of treatment, psoriasis is still a challenge 
for doctors and a burden for patients. The recent discovery of IL-17, which contributes to development of psoriasis, opened new possibilities for further treatment modalities. In January 2015, secukinumab was approved for the treatment of adults with moderate-to-severe plaque psoriasis. Two anti-IL-7 inhibitors, brodalumab and ixekizumab, are currently in phase III clinical trials (as of February 2015). A novel approach is the development of bi-specific antibodies, which target both IL-17 and TNF- $\alpha$. The preliminary results show significant efficacy and a high safety profile of biological drugs in clinical trials. However, more studies must be conducted for further elucidation of the long-term treatment using this group of agents.

\section{Conflict of interest}

The authors declare no conflict of interest.

\section{References}

1. Gelfand JM, Weinstein R, Porter SB, et al. Prevalence and treatment of psoriasis in the United Kingdom: a populationbased study. Arch Dermatol 2005; 141: 1537-41.

2. Papp K, Berth-Jones J, Kragballe K, de la Brassinne M. Scalp psoriasis: a review of current topical treatment options. J Eur Acad Dermatol Venereol 2007; 21: 1151-60.

3. Augustin M, Radtke MA. Quality of life in psoriasis patients. Expert Rev Pharmacoecon Outcomes Res 2014; 14: 559-68.

4. Richards HL, Fortune DG, Main CJ, Griffiths CE. Stigmatization and psoriasis. Br J Dermatol 2003; 149: 209-11.

5. Gaffen SL. Recent advances in the IL-17 cytokine family. Curr Opin Immunol 2011; 23: 613-9.

6. Lønnberg AS, Zachariae C, Skov L. Targeting of interleukin-17 in the treatment of psoriasis. Clin Cosmet Investig Dermatol 2014; 7: 251-9.

7. Girolomoni G, Mrowietz U, Paul C. Psoriasis: rationale for targeting interleukin-17. Br J Dermatol 2012; 167: 717-24.

8. Martin DA, Towne JE, Kricorian G, et al. The emerging role of IL-17 in the pathogenesis of psoriasis: preclinical and clinical findings. J Invest Dermatol 2013; 133: 17-26.

9. Adami S, Cavani A, Rossi F, Girolomoni G. The role of interleukin-17A in psoriatic disease. BioDrugs 2014; 28: 487-97.

10. Gaffen SL. Structure and signalling in the IL-17 receptor family. Nat Rev Immunol 2009; 9: 556-67.

11. Chiricozzi A, Krueger JG. IL-17 targeted therapies for psoriasis. Expert Opin Investig Drugs 2013; 22: 993-1005.

12. Yang J, Sundrud MS, Skepner J, Yamagata T. Targeting Th17 cells in autoimmune diseases. Trends Pharmacol Sci 2014; 35: 493-500.

13. Shabgah AG, Fattahi E, Shahneh FZ. Interleukin-17 in human inflammatory diseases. Postep Derm Alergol 2014; 31: 256-61.

14. Chen S, Crother TR, Arditi M. Emerging role of IL-17 in atherosclerosis. J Innate Immun 2010; 2: 325-33.

15. Nwe SM, Champlain AH, Gordon KB. Rationale and early clinical data on IL-17 blockade in psoriasis. Expert Rev Clin Immunol 2013; 9: 677-82.

16. Coimbra S, Figueiredo A, Santos-Silva A. Brodalumab: an evidence-based review of its potential in the treatment of moderate-to-severe psoriasis. Core Evid 2014; 9: 89-97.
17. Lynde CW, Poulin Y, Vender R, et al. Interleukin 17A: toward a new understanding of psoriasis pathogenesis. J Am Acad Dermatol 2014; 71: 141-50.

18. Rich P, Sigurgeirsson B, Thaci D, et al. Secukinumab induction and maintenance therapy in moderate-to-severe plaque psoriasis: a randomized, double-blind, placebo-controlled, phase II regimen-finding study. Br J Dermatol 2013; 168: 402-11.

19. Langley RG, Elewski BE, Lebwohl M, et al. Secukinumab in plaque psoriasis: results of two phase 3 trials. N Engl J Med 2014; 371: 326-38.

20. Papp KA, Langley RG, Sigurgeirsson B, et al. Efficacy and safety of secukinumab in the treatment of moderate-to-severe plaque psoriasis: a randomized, double-blind, placebocontrolled phase II dose-ranging study. Br J Dermatol 2013; 168: 412-21.

21. Blauvelt A, Prinz JC, Gottlieb AB, et al. Secukinumab administration by pre-filled syringe: efficacy, safety, and usability results from a randomized controlled trial in psoriasis (FEATURE). Br I Dermatol 2015; 172: 484-93.

22. Russell CB, Rand H, Bigler J, et al. Gene expression profiles normalized in psoriatic skin by treatment with brodalumab, a human anti-IL-17 receptor monoclonal antibody. J Immunol 2014; 192: 3828-36.

23. Papp KA, Reid C, Foley P, et al. Anti-IL-17 receptor antibody AMG 827 leads to rapid clinical response in subjects with moderate to severe psoriasis: results from a phase I, randomized, placebo-controlled trial. J Invest Dermatol 2012; 132: 2466-9.

24. Papp KA, Leonardi C, Menter A, et al. Brodalumab, an antiinterleukin-17-receptor antibody for psoriasis. N Engl J Med 2012; 366: 1181-9.

25. Mease PJ, Genovese MC, Greenwald MW, et al. Brodalumab, an anti-IL17RA monoclonal antibody, in psoriatic arthritis. N Engl J Med 2014; 370: 2295-306.

26. Lebwohl M, Strober B, Menter A, et al. Phase 3 studies comparing brodalumab with ustekinumab in psoriasis. N Engl J Med 2015; 373: 1318-28.

27. Ren $\mathrm{V}$, Dao H. Potential role of ixekizumab in the treatment of moderate-to-severe plaque psoriasis. Clin Cosmet Investig Dermatol 2013; 6: 75-80.

28. Krueger JG, Fretzin S, Suárez-Farińas M, et al. IL-17A is essential for cell activation and inflammatory gene circuits in subjects with psoriasis. J Allergy Clin Immunol 2012; 130: 145-54.e9.

29. Leonardi C, Matheson R, Zachariae C, et al. Anti-interleukin-17 monoclonal antibody ixekizumab in chronic plaque psoriasis. N Engl J Med 2012; 366: 1190-9.

30. Zhu B, Edson-Heredia E, Cameron GS, et al. Early clinical response as a predictor of subsequent response to ixekizumab treatment: results from a phase II study of patients with moderate-to-severe plaque psoriasis. Br I Dermatol 2013; 169: 1337-41.

31. Gordon KB, Leonardi CL, Lebwohl M, et al. A 52-week, openlabel study of the efficacy and safety of ixekizumab, an antiinterleukin-17A monoclonal antibody, in patients with chronic plaque psoriasis. J Am Acad Dermatol 2014; 71: 1176-82.

32. Miossec P, Kolls JK. Targeting IL-17 and Th17 cells in chronic inflammation. Nat Rev Drug Discov 2012; 11: 763-76.

33. Kurzeja M, Rudnicka L, Olszewska M. New interleukin-23 pathway inhibitors in dermatology: ustekinumab, briakinumab, and secukinumab. Am J Clin Dermatol 2011; 12: 113-25.

34. Lu R, Zeng X, Han Q, et al. Overexpression and selectively regulatory roles of IL-23/IL-17 axis in the lesions of oral lichen planus. Mediators Inflamm 2014; 2014: 701094. 
35. Tanemura A, Oiso N, Nakano M, et al. Alopecia areata: infiltration of Th17 cells in the dermis, particularly around hair follicles. Dermatology 2013; 226: 333-6.

36. Ohtsuka M, Yamamoto T. Rare association of pyoderma gangrenosum and palmoplantar pustulosis: a case report and review of the previous works. J Dermatol 2014; 41: 732-5.

37. Gaowa S, Zhou W, Yu L, et al. Effect of Th17 and Treg axis disorder on outcomes of pulmonary arterial hypertension in connective tissue diseases. Mediators Inflamm 2014; 2014: 247372.

38. Xue J, Su W, Chen Z, et al. Overexpression of interleukin-23 and interleukin-17 in the lesion of pemphigus vulgaris: a preliminary study. Mediators Inflamm 2014; 2014: 463928

39. Zebrowska A, Wagrowska-Danilewicz M, Danilewicz M, et al. IL-17 expression in dermatitis herpetiformis and bullous pemphigoid. Mediators Inflamm 2013; 2013: 967987.

40. Batista DI, Perez L, Orfali RL, et al. Profile of skin barrier proteins (filaggrin, claudins 1 and 4) and Th1/Th2/Th17 cytokines in adults with atopic dermatitis. J Eur Acad Dermato Venereol 2015; 29: 1091-5.

41. Luo Z, Wang H, Wu Y, et al. Clinical significance of IL-23 regulating IL-17A and/or IL-17F positive Th17 cells in chronic periodontitis. Mediators Inflamm 2014; 2014: 627959. 